\title{
Target-Dependent Sexual Differentiation of a Limbic-Hypothalamic Neural Pathway
}

\author{
Maria A. Ibanez, ${ }^{1}$ Guibao Gu, ${ }^{1}$ and Richard B. Simerly ${ }^{1,2}$ \\ ${ }^{1}$ Division of Neuroscience, Oregon Regional Primate Research Center, Beaverton, Oregon 97006, and 2Program in \\ Neuroscience, Oregon Health and Sciences University, Portland, Oregon 97201
}

Neural pathways between sexually dimorphic forebrain regions develop under the influence of sex steroid hormones during the perinatal period, but how these hormones specify precise sexspecific patterns of connectivity is unknown. A heterochronic coculture system was used to demonstrate that sex steroid hormones direct development of a sexually dimorphic limbichypothalamic neural pathway through a target-dependent mechanism. Explants of the principal nucleus of the bed nuclei of the stria terminalis (BSTp) extend neurites toward explants of the anteroventral periventricular nucleus (AVPV) derived from male but not female rats. Coculture of BSTp explants from male rats with AVPV explants derived from females treated in vivo with testosterone for $9 \mathrm{~d}$ resulted in a high density of neurites extending from the BSTp to the AVPV explant, as was the case when the BSTp explants were derived from females and the AVPV explants were derived from males or androgen-treated females. These in vitro findings suggest that during the postnatal period testosterone induces a target-derived, diffusible chemotropic activity that results in a sexually dimorphic pattern of connectivity.

Key words: sexual differentiation; axonal guidance; anteroventral periventricular nucleus; bed nucleus of the stria terminalis; coculture; preoptic region
Forebrain neural pathways that control reproduction are different in male and female mammals and show considerable plasticity throughout life. Despite the adaptive significance of developmental processes that promote reproduction, little is known about the underlying mechanisms controlling development of the sexually dimorphic forebrain circuits mediating gonadotropin secretion and copulatory behavior. It is well established that the high levels of testosterone present in neonatal males exert a powerful influence on neuronal survival and development of connectivity (Raisman and Field, 1971; Arai, 1981; Arnold and Gorski, 1984; Arnold and Jordan, 1988; Simerly, 1999; Toran-Allerand et al., 1999). However, because there is a great deal of interconnectivity between sexually dimorphic nuclei that express high levels of sex steroid receptors during development, it is difficult to identify the sites of action for testosterone in determining sex-specific patterns of forebrain neural circuitry. Thus, it is unknown whether testosterone acts directly on projection neurons in sexually dimorphic nuclei to promote extension of growth cones and branching of axons or directs development of dimorphic patterns of connectivity by inducing expression of target-derived factors that preferentially guide axons to their targets.

To more clearly define the mechanisms that govern development of sexually dimorphic pathways, we focused our attention on a sexually dimorphic, limbic-hypothalamic pathway in the rodent forebrain that has several unique features. The projection from

Received Feb. 16, 2001; revised May 7, 2001; accepted May 18, 2001.

This work was supported by National Institutes of Health Grants NS37952 and RR00163. We thank Drs. R. Robertson, S. Wray, and D. Toran-Allerand for essential advice on organotypic coculture methods and Dr. A. Cornea for advice on image analysis. We are also grateful to Danielle Mitrakul for preparation of this manuscript and to Drs. S. R. Ojeda, M. S. Smith, and E. R. Spindel for comments on this manuscript.

Correspondence should be addressed to Richard B. Simerly, Oregon Regional Primate Research Center, 505 Northwest 185th Avenue, Beaverton, OR 97006. E-mail: simerlyr@OHSU.edu.

Copyright (C) 2001 Society for Neuroscience 0270-6474/01/215652-08\$15.00/0 the principal nucleus of the bed nuclei of the stria terminalis (BSTp) to the anteroventral periventricular nucleus of the preoptic region (AVPV) is the most robust sexually dimorphic pathway yet identified (Hutton et al., 1998). The BSTp is larger in males and functions as part of a sexually dimorphic limbic pathway that relays olfactory information to the hypothalamus (Simerly, 1990; Segovia and Guillamón, 1993). It provides strong projections to sexually dimorphic parts of the hypothalamus, including the AVPV, which plays a critical role in the control of gonadotropin secretion and ovulation (Wiegand and Terasawa, 1982; Simerly, 1995). In contrast to the BSTp, the AVPV is larger in female rats and contains a greater number of dopaminergic and peptidergic neurons (Bleier et al., 1982; Simerly, 1995). Despite its smaller size in males, the AVPV receives a massive innervation from the BSTp that is $\sim 10$-fold greater than the homologous input in females, and this sex difference is determined by exposure to testosterone during the postnatal period ( $\mathrm{Gu}$ and Simerly, 1997). Moreover, this pathway develops during the second week of life in a sexually dimorphic pattern that suggests a directed mechanism of axon guidance; fibers appear to extend from the BSTp in both neonatal male and female rats, but only in males does a high density of fibers reach the AVPV (Hutton et al., 1998). Thus, the projection from the BSTp to the AVPV represents a sexually dimorphic pathway between two regions with divergent developmental histories: exposure to sex steroids increases the number of cells in the BSTp, yet has the opposite effect on AVPV neurons, resulting in a massive convergence of information relayed by the BSTp onto AVPV neurons. However, testosterone could influence the differentiation of this pathway by acting on either the BSTp or AVPV, because neurons in both regions express high levels of receptors for sex steroid hormones during the first week of life (DonCarlos and Handa, 1994; McAbee and DonCarlos, 1998; Simerly, 1999). In the present study we used a heterochronic organotypic coculture system to 
determine whether the development of the projection from the BSTp to the AVPV is a sex-specific property of BSTp neurons or is dependent on the neonatal hormone environment in which the AVPV develops.

\section{MATERIALS AND METHODS}

Preparation of cocultures and tissue preparation. Timed pregnant Sprague Dawley rats were obtained from B\&K Universal (Kent, WA) and observed until birth of litters [designated postnatal day $0(\mathrm{P} 0)$ ]. Pairs of neonates were decapitated on P5 (for BSTp explants) and P9 (for AV PV explants), and the brains were quickly removed and placed in an ice-cold Petri dish for gross dissection. These ages were chosen because the projection fibers from the BSTp normally reach the AVPV in male rats on P9 in vivo (Hutton et al., 1998). Moreover, the results of preliminary coculture experiments indicated that the density of neurites that reached the AVPV explant is enhanced when the AVPV explant is taken from a $\mathrm{P} 9$ rat than if it is derived from a younger [P5-P8 or older (P10-P12) animal]. We also found that neurite outgrowth from BSTp explants was more robust if they are derived from P5 pups compared with those taken from neonates on later postnatal days (P7-P10).

Fully androgen-sterilized female rats (Barraclough, 1979; Gerall and Givon, 1992) were prepared by implanting subcutaneous pellets of testosterone $(0.5 \mathrm{mg}$; Innovative Research of America, Toledo, $\mathrm{OH}$ ) within $2 \mathrm{hr}$ after birth. These pellets are designed to provide stable levels of circulating testosterone for up to 3 weeks.

Each brain was blocked and submerged in cold Geys balanced salt solution for sectioning at $200 \mu \mathrm{m}$ on a vibrating tissue slicer (Electron Microscopy Sciences, Fort Washington, PA). Slices were then transferred to six-well plates containing cold EOL-1 medium (Annis et al., 1990) until microdissection of explants. Explants of the BSTp and AVPV were prepared by microdissecting each region under a stereo microscope. The AVPV explant included the bilateral nuclei (separated by the third ventricle) and was limited dorsally by the top of the third ventricle. The accuracy of the microdissection was confirmed by labeling AV PV neurons in isolated explants for tyrosine hydroxylase (Ibanez et al., 1998) or estrogen receptor mRNA and protein (E. M. Waters and R. B. Simerly, unpublished observations), which are established markers for AVPV neurons in vivo. The BSTp explant was dissected from either the right or left side of the brain of a different animal (P5) by making two intersecting cuts directed ventrally from the lateral ventricle that bordered the medial and lateral edges of the BSTp and then trimming off the dorsal edge of the explant. That this procedure produced specific BSTp explants was confirmed by using in situ hybridization histochemistry to visualize neurons that express markers for the BSTp (estrogen receptor, androgen receptor, and preprocholecystokinin mRNAs) in isolated explant cultures (Hutton and Simerly, 1997; L. A. Hutton and R. B. Simerly, unpublished observations).

Cocultures composed of BSTp and AVPV explants were prepared according to the method of Guthrie and Lumsden (1994) with minor modifications, as described by Kuang et al. (1994). The two explants were positioned onto poly-D-lysine-coated coverslips by placing them above the coverslips in a suspension of rat tail collagen in deficient Ham's F-12 media. An additional $15 \mu \mathrm{l}$ of the collagen suspension was added on top of the explants, and they were oriented with the medial side of the BSTp explant adjacent to, and $0.5-0.8 \mathrm{~mm}$ from, the lateral edge of the AVPV explant. The coverslips were then placed into a $35.6^{\circ} \mathrm{C}$ humidified incubator with $5 \% \mathrm{CO}_{2}$ for $2 \mathrm{hr}$ to allow the collagen to polymerize. After collagen polymerization, the coverslips were placed in flat-bottom tubes containing $0.9 \mathrm{ml}$ of EOL-1 serum-free medium (Annis et al., 1990) containing $10^{-7} \mathrm{M}$ testosterone, returned to the incubator, and left stationary for $10 \mathrm{~d}$. The orientation of the coverslips in the tubes was varied to randomize effects of tissue placement within tubes. On the fifth day in vitro, an additional $90 \mu \mathrm{l}$ of EOL-1 medium was added to each tube. Testosterone was included in the medium for all of the cocultures, because we determined that it increased the density of neurites between explants approximately fivefold. However, if testosterone was omitted from the media, similar results were obtained with respect to the sexually dimorphic target-dependent formation of neurites extending from the BSTp to the AVPV.

DiI Labeling and immunohistochemistry. After $10 \mathrm{~d}$ in vitro, cocultures were fixed with $4 \%$ paraformaldehyde and kept at $4^{\circ} \mathrm{C}$ until labeled with the lipophilic fluorescent tracer DiI (Godement et al., 1987). With the aid of a stereo microscope, small crystals of DiI were placed into the center of the BST explant of each coculture and maintained in $4 \%$ paraformal- dehyde at $37^{\circ} \mathrm{C}$ for $24 \mathrm{hr}$. After labeling with DiI, the cocultures were stored at $4^{\circ} \mathrm{C}$ until analysis. Before evaluation, the cocultures were labeled with the nuclear stain Hoechst 33258 (Molecular Probes, Eugene, OR) by incubation in a solution of the stain $(3 \mu \mathrm{g} / \mathrm{ml})$ for $5 \mathrm{~min}$ at room temperature. The coverslips were rinsed in potasium phosphate-buffered saline (KPBS), placed explant-down onto a cavity microscope slide containing a drop of glycerol mountant, and then examined by using conventional and confocal microscopy.

For immunohistochemical labeling, the cocultures were fixed in $4 \%$ paraformaldehyde for $1 \mathrm{hr}$ at $4^{\circ} \mathrm{C}$. The cocultures were then pretreated overnight in a blocking solution (LKPBS) containing 5\% normal goat serum and $1 \%$ Triton $\mathrm{X}-100$ at $4^{\circ} \mathrm{C}$ in sodium phosphate-buffered saline. The cocultures were then transferred to a mixture of primary antibodies diluted in LKPBS [1:1000 for mouse anti-GAP43 (Chemicon, Temecula, CA) and mouse anti-MAP2 (Sigma, St. Louis, MO) and 1:1500 for mouse anti- $\beta$-III-tubulin (Babco, Berkeley, CA) and 1:2000 for rabbit anti-GFAP (Chemicon)] and incubated at $4^{\circ} \mathrm{C}$ for $72 \mathrm{hr}$. Cocultures were transferred to a mixture of secondary antibodies diluted in LKPBS [1:200 for goat anti-rabbit IgG conjugated with FITC and donkey antimouse IgG conjugated with Rd-redX (both from Jackson ImmunoResearch, West Grove, PA) or goat anti-mouse IgG conjugated with Alexa 488 and goat anti-rabbit IgG conjugated with Alexa 594 (both from Molecular Probes)] and incubated for $1 \mathrm{hr}$ with gentle agitation at room temperature. Cocultures were then examined by conventional and confocal microscopy.

Analysis and quantification. To prepare the images presented in the figures, stacks of 32 adjacent confocal images were collected through a 6to $8-\mu \mathrm{m}$-thick region through the center of the cocultures by using a $25 \times$ oil immersion objective [numerical aperture (NA) 0.75, UV corrected] and a Leica (Nussloch, Germany) SP confocal microscope configured to optimally image red fluorescence, followed by sequential collection of a series of corresponding images obtained using UV excitation. Projection images were prepared for each pair of image stacks and combined into a single pseudocolor image by using MetaMorph (Universal Imaging Corp., West Chester, PA) image analysis software. For the semiquantitative comparison of neurite density in heterochronic cocultures (see Fig. 4 ), image stacks were collected at $1 \mu \mathrm{m}$ intervals through the entire thickness of each coculture $(50-136 \mu \mathrm{m})$ by using a $63 \times$ water-corrected objective (NA 1.2). Two volumes were sampled for each coculture: one adjacent to the leading edge of the BSTp explant (box A) and the other adjacent to the edge of the AVPV explant (box B).

Each stack of images was analyzed by using MetaMorph image analysis software (Universal Imaging Corp.), and the density of labeled neurites was estimated according to the following procedure. Each image plane was binarized according to threshold criteria to isolate labeled fibers from background, as well as to compensate for differences in fluorescence intensity, and then skeletonized so that each fiber segment was 1 pixel thick. The integrated intensity was then calculated for each image, which reflects the total number of pixels in the skeletonized image and is proportional to the total length of labeled fibers in the image. This procedure was performed sequentially on each image plane in the stack, and the results were ported to a data spreadsheet. The pixel densities for all image planes in a stack were summed, and the resulting value was taken as an index of fiber density in the volume sampled. Three cocultures for each experimental pairing of BSTp and AVPV explants were analyzed, and the Kruskal-Wallis ANOVA, with a Fisher's least significant difference post hoc comparison, was used to define significant differences between experimental groups.

\section{RESULTS}

\section{Heterochronic cocultures of the BSTp and AVPV}

Cocultures consisting of AVPV explants derived from male neonatal rats on P9 and BSTp explants dissected from the brains of male rats on P5 were prepared and maintained under defined conditions in vitro. On the 10th day in vitro, each coculture was labeled with DiI, and high densities of DiI-labeled fibers extended from the BSTp to the AVPV explants in the male-male cocultures. The density of fibers directed toward the AVPV appeared to be substantially greater than that from the opposite side of the BSTp (Fig. 1). These fibers appear to be axons, because they contain GAP43 and $\beta$-III-tubulin-immunoreactivity (McGuire et al., 1988; Lee et al., 1990; Dani et al., 1991; Moody et al., 1996) 


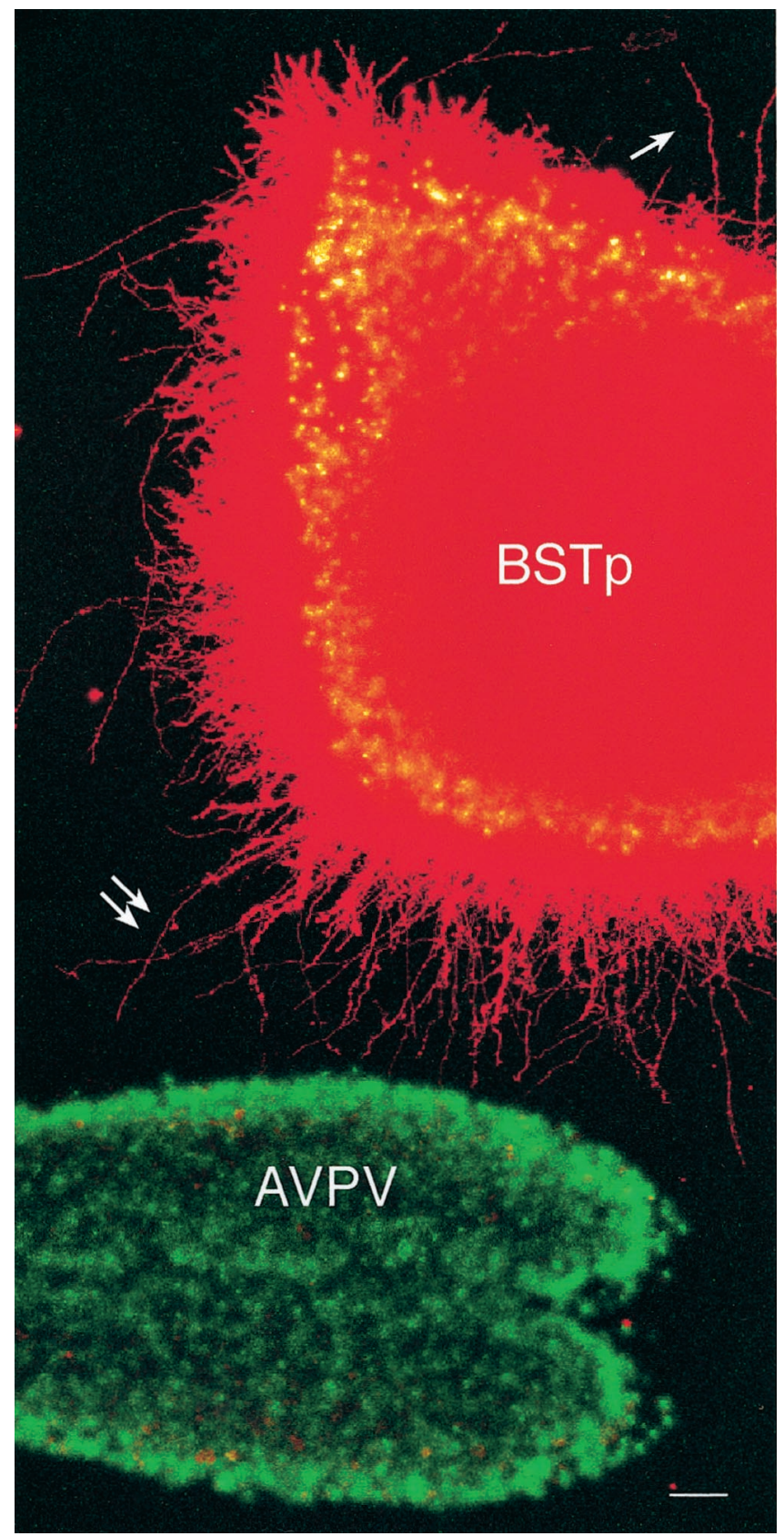

Figure 1. Heterochronic cocultures of the BSTp and AVPV. A montage of two low-magnification confocal projection images (imaged using a $10 \times$ multi-immersion objective; NA 0.4) of a BST-AVPV coculture (both explants derived from male rats) shows the relationship between the DiI-labeled BSTp explant and the AVPV explant (stained with Hoechst 33258 ) in a representative experiment. Note the greater density of DiIlabeled neurites extending from the BSTp explant toward the AVPV explant (double arrow) compared with the number of neurites on the opposite side of the BSTp explant (single arrow). Scale bar, $40 \mu \mathrm{m}$.

but do not express detectable levels of glial or dendritic markers (Fig. 2). Neurites extending from the BSTp toward the AVPV explant were not immunoreactive for the glial marker GFAP (Rinaman et al., 1993) or for MAP2, which is expressed primarily in neuronal cell bodies and dendrites (Pennypacker et al., 1991; Shafit-Zagardo and Kalcheva, 1998). Substitution of the AVPV explants with tissue derived from cerebral cortex or caudoputamen did not result in any detectable fibers extending from the
BSTp, nor were any fibers observed extending from the BSTp explant to an explant derived from the arcuate nucleus of the hypothalamus, which does receive an input from the BSTp in adulthood, demonstrating a high degree of specificity for the BSTp-AVPV coculture system.

\section{Target-dependent induction of neurites in heterochronic cocultures}

In contrast to the high density of DiI-labeled fibers observed in male-male cocultures, only a few labeled neurites were detected when the cocultures were prepared with BSTp explants from males and AVPV explants from females (Fig. 3). Although there was no significant difference in the density of DiI-labeled fibers near the BSTp explant, the density of labeled fibers extending as far as the edge of the AVPV explant in the male-female cocultures was $\sim 1 / 10$ that of the male-male cocultures (Fig. 4). Thus, the sexually dimorphic development of projections from the BSTp to the AVPV appears to occur between cocultured explants as it does in vivo and is dependent on whether the AVPV target is derived from a male or a female rat.

Exposing female rats to testosterone during the postnatal period results in a fully androgen-sterilized (FAS) animal that is unable to mount preovulatory gonadotropin surges (Harris, 1970; Gorski, 1973; Barraclough, 1979; Gerall and Givon, 1992). To determine whether the sex difference in the density of fibers extending from the BSTp to the AVPV is attributable to the action of sex steroid hormones on the AVPV, we prepared cocultures from AVPV explants derived from female rats that received testosterone implants on the day of birth combined with BSTp explants derived from intact males (P5). Continuous exposure of female neonates to testosterone during the first 9 postnatal days in vivo (before preparing the cocultures) caused a dramatic masculinization of the male-female cocultures (Fig. 3C). Cocultures prepared with BSTp explants derived from P5 males and AVPV explants from FAS females had a similar density of labeled neurites extending from the BSTp to the AVPV explant as did male-male cocultures (Fig. 4). Thus, the development of projections from the BSTp to the AVPV appears to be dependent primarily on whether the target tissue was exposed to testosterone during the postnatal period.

\section{Target-dependent induction of neurite outgrowth from BSTp explants derived from female rats}

Although the results of the previous experiments argue strongly that the primary factors controlling the density of BSTp projections to the AVPV are target-dependent, they do not address the possible role of the BSTp in mediating this hormone-directed pattern of development. To determine whether the development of projections from the BSTp to the AVPV is independent of the hormonal history of the BSTp, we prepared cocultures composed of BSTp explants derived from female rats (P5) and AVPV explants derived from intact male rats (P9). The density of labeled neurites extending from the BSTp to the AVPV in these female-male cocultures was similar to that in either male-male or male-FAS cocultures (Figs. 4, 5A). However, if the AVPV explant was derived from a P9 female rat, very few labeled fibers were found to extend toward the target (Figs. 4, 5B) suggesting that the failure of male BSTp explants to extend neurites to female AVPV explants is primarily dependent on the sex of the animal contributing the AVPV explant.

To determine whether exposure of the AVPV to testosterone during postnatal life is sufficient to induce extension of neurites 

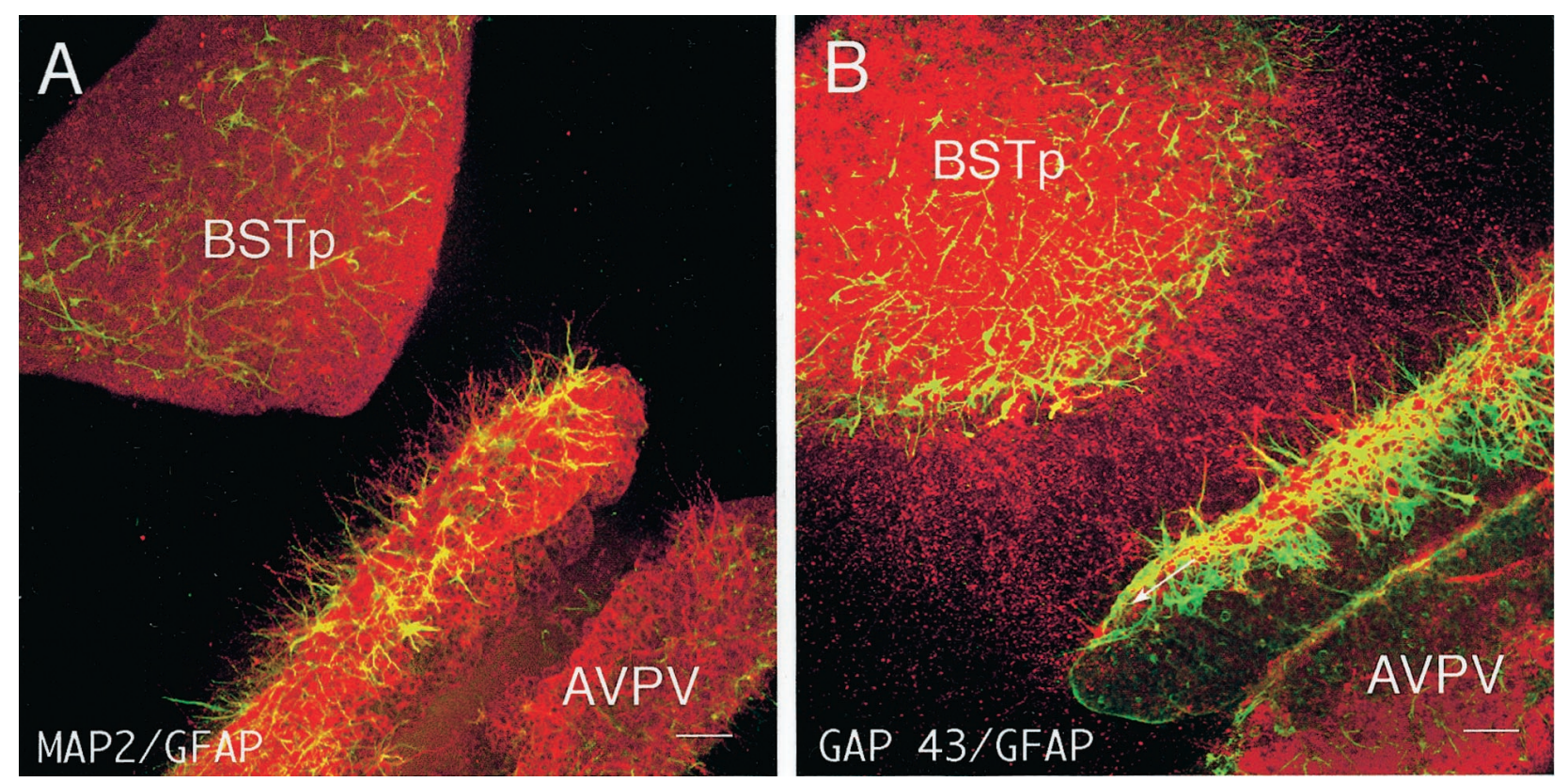

Figure 2. BSTp neurites contain GAP-43 immunoreactivity but not GFAP or MAP2. Dual immunofluorescence images show localization of glial and neuronal markers in a BSTp-AVPV coculture. A, Neurites extending from the BSTp explant to the AVPV explant lack the glial marker GFAP (green) or immunoreactivity for MAP2 (red), which is expressed primarily in neuronal cell bodies and dendrites. Note robust expression of GFAP and MAP2 in the proximal side of the AVPV explant. $B$, In contrast, neurites between the cocultured explants were immunoreactive for GAP43 (red), which is expressed in developing axons. Scale bars, $40 \mu \mathrm{m}$.
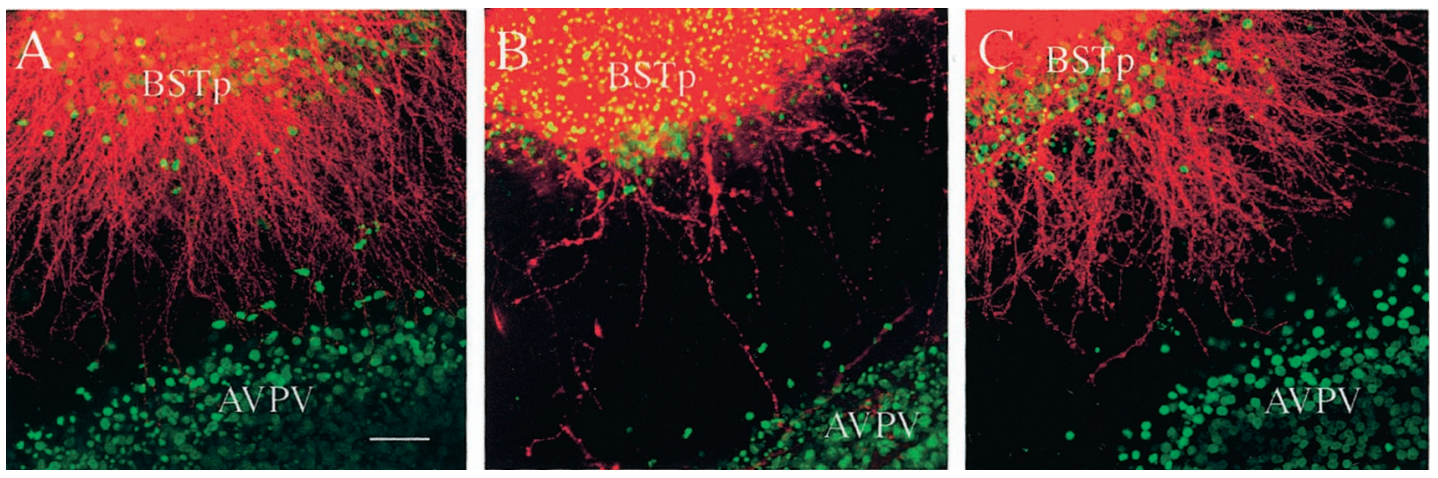

Figure 3. Target-dependent induction of neurites in heterochronic cocultures. Confocal image projections of BSTp-AVPV cocultures show DiI labeling of neurites (pseudocolored red) that extend from the BSTp toward the AVPV. The BSTp explant was derived from a male rat on P5 and cocultured with an AVPV explant (stained with Hoechst 33258 nuclear stain and pseudocolored green) derived from a P9 male $(A)$, female $(B)$, or androgen-treated female $(C)$ rat. A dramatic difference in the density of neurites between the cocultured explants was observed, which depended on whether the AVPV explant was derived from a male $(A)$ or female $(B)$ rat. Treatment of neonatal female rats with testosterone during the first $9 \mathrm{~d}$ of life in vivo masculinized the appearance of the cocultures $(C)$, suggesting that the target-dependent formation of neurites extending from the BSTp to the AVPV is determined by exposure to sex steroid hormones during the neonatal period. Scale bar, $15 \mu \mathrm{m}$.

from BSTp explants to AVPV target tissue, we prepared cocultures consisting of BSTp explants derived from normal female rats and AVPV explants derived from P9 female rats that had received implants of testosterone on the day of birth. These cocultures showed the masculine pattern of neurite extension from the BSTp explant (Fig. 5C) and the density of fibers reaching the AVPV target was remarkably similar to that of male-male and female-male cultures (Fig. 4). Taken together, these findings indicate that the development of projections from the BSTp to the AVPV is dependent on exposure of the target tissue to testosterone during the first 9 postnatal days and is independent of the hormone history of the BSTp neurons.

\section{DISCUSSION}

The ontogeny of the BSTp-to-AVPV pathway has been studied in vivo, and the results indicated that it develops according to a directed mechanism with marked target specificity. Implants of DiI into the BSTp of P10 rats labeled a dense plexus of fibers in the AVPV of males, yet in females the AVPV contained only a few labeled fibers at all ages examined (Hutton et al., 1998). Thus the BSTp-to-AVPV pathway does not form in both sexes and then regress in female rats but apparently develops only in males. In addition, fibers appear to extend out of the BSTp in both male and female rats during development, but only in males is there a 

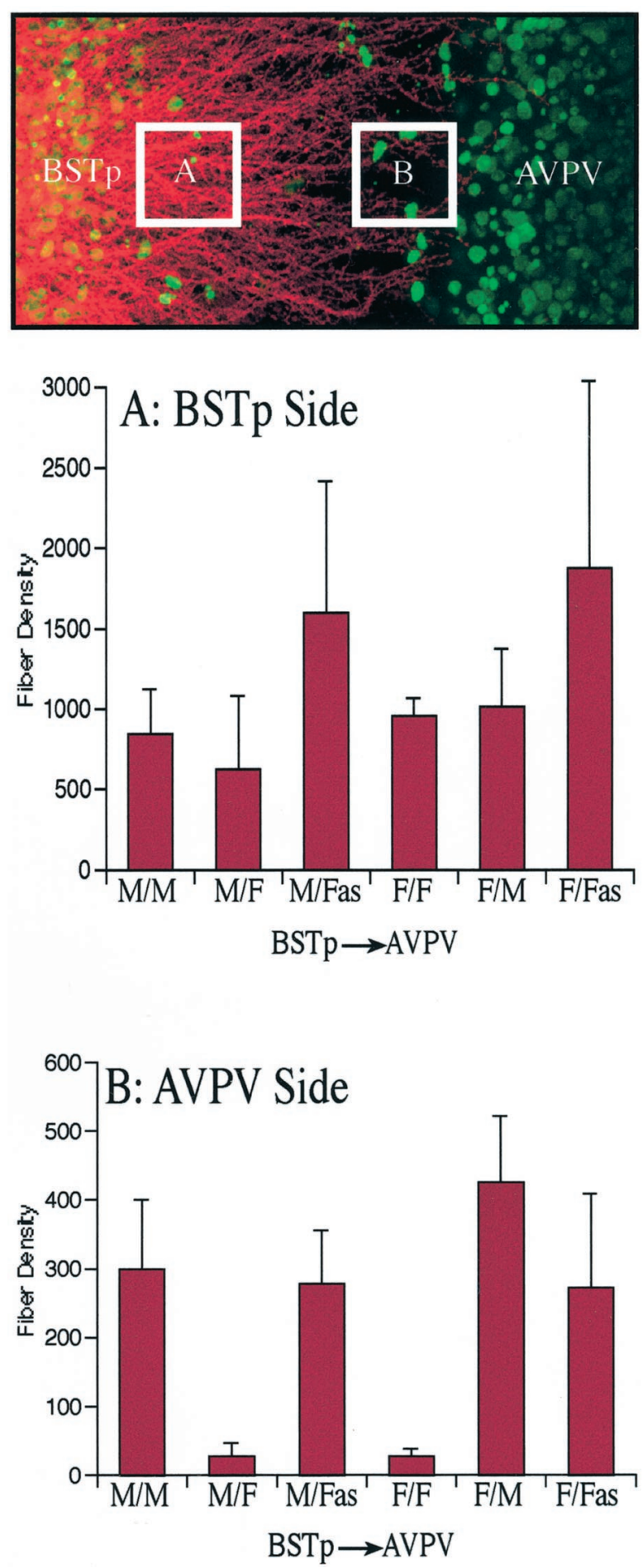

Figure 4. Preference of BSTp axon growth. Semiquantitative comparison of neurite density is shown in cocultures with BSTp explants taken from male or female neonates and AVPV targets derived from male, female, or androgen-treated female rats. Heterochronic cocultures were prepared and labeled with DiI as described in Figure 1. Two volumes were sampled for each coculture: one adjacent to the leading edge of the BSTp explant (box $A$ ) and the other adjacent to the edge of the AVPV explant (box B). The columns in the graphs represent mean fiber densities. Error bars indicate SEM. Fiber density was confirmed to be significantly lower $(p \leq 0.008)$ in the male-female $(M / F)$ and female-female $(F / F)$ cocultures than in the experimental pairings that had AVPV explants derived from male $(M / M, F / M)$ or testosterone-treated female (M/Fas, F/Fas) rats. dense plexus of BSTp terminals in the AVPV, suggesting that the sex difference in the density of projections to the AVPV is not simply attributable to the greater number of neurons in the BSTp of males. Our in vitro findings support these conclusions and for the first time provide direct evidence for target-dependent development of a sexually dimorphic forebrain pathway.

Although it is logical that the cocultures work best if the AVPV target is derived from animals that are approximately the same age as those in which the AVPV is innervated by the BSTp in vivo, it is unclear why the optimal age for the BSTp explant is P5. In reconstituting this sexually dimorphic pathway in vitro, it is perhaps more important for the BSTp explant to match the age during which there is maximal outgrowth from the BSTp in vivo rather than the age at which the axons reach their targets. Our results also argue that the AVPV is the site of action for testosterone in directing the sexual differentiation of the BSTp to AVPV projection, because a high density of neurites reached the AVPV only in cocultures composed of AVPV explants derived from male or androgen-treated female rats, regardless of the source of the BSTp explant. Only a few neurites reach the AVPV target when cocultures include AVPV explants taken from intact female rats. In contrast, cocultures consisting of BSTp explants derived from either male or female rats and AV PV explants taken from males or androgen-treated females display a high density of fibers extending between the two explants. Together, these results demonstrate that the ability of the BSTp to provide a high density of neurites that extend to the AVPV is dependent on exposure of the AVPV to testosterone.

Because the testosterone treatments were performed in vivo before making the cocultures, it is possible that the effects of hormone exposure were conveyed trans-synaptically from other hormone-sensitive regions that provide afferents to the AVPV (Simerly, 1998). Such indirect hormonal regulation of development seems unlikely, because many of the major afferents to the AVPV, such as those from the arcuate and dorsomedial hypothalamic nuclei, have not formed by P9 (S. Draper and R. B. Simerly, unpublished observations). Alternatively, because estrogen and androgen receptors are expressed in abundance in BSTp explants (Hutton and Simerly, 1997), the testosterone present in the medium could influence the responsiveness of BSTp neurons to AVPV-derived factors. In fact, we did observe that addition of testosterone to the medium increased the density of fibers between cocultured explants in all cocultures, yet could not abolish the AVPV-dependent sex difference. Testosterone action on the BSTp during the period in vitro cannot explain the results of the coculture experiments, because testosterone is present in the medium in all experiments and did not cause a high density of neurite extension in the male-female or female-female cocultures.

The AVPV is larger and contains more neurons in female rats, including sexually dimorphic populations of neurons that contain various neurotransmitter substances (Bleier et al., 1982; Bloch and Gorski, 1988; Sumida et al., 1993). With few exceptions, these neurons are more numerous in females, and their numbers are reduced by exposure to testosterone (Simerly, 1995, 1999). Thus, exposure of newborn female rats to testosterone appears to reduce the number of neurons that mature in the AVPV but to promote the innervation of the remaining neurons. It is a common observation that early exposure to testosterone alters the number of neurons that mature in sexually dimorphic nuclei (Forger et al., 1992; Tobet and Fox, 1992; Cooke et al., 1998; Simerly, 1999). If testosterone increases the number of neurons in 

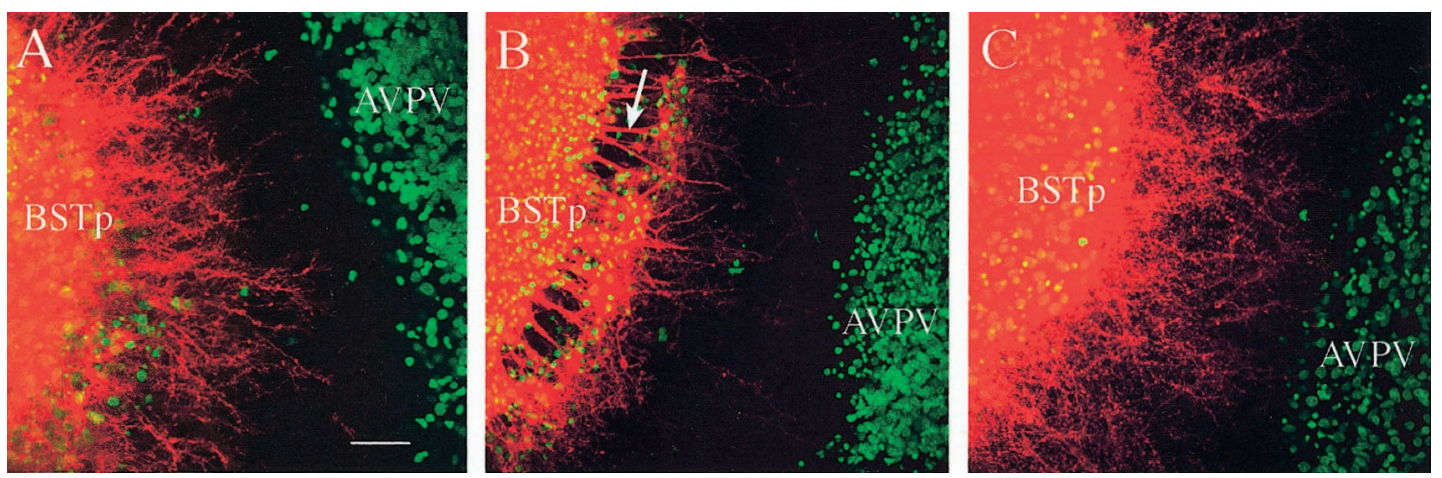

Figure 5. Target-dependent induction of neurite outgrowth from BSTp explants derived from female rats. Heterochronic cocultures were prepared as in Figure 2, except that in each case the BSTp explant was derived from a female rat on P5. BSTp explants were cocultured with an AVPV explant (stained with Hoechst 33258 nuclear stain and pseudocolored green) derived from a P9 male $(A)$, female $(B)$, or androgen-treated female $(C)$ rat. DiI was used to label neurites extending from the BSTp explant (pseudocolored red), and confocal image projections of the cocultures were obtained as described in Figure 4. A, A high density of labeled neurites extend from a BSTp explant, taken from a female rat on P5, toward an AVPV explant derived from a male rat on P9, as they do in male-male cocultures (see Fig. 4). B, There are remarkably few labeled neurites in the space between the explants if both the BSTp and AVPV explants are derived from female rats. Although all explants were positioned $\sim 0.5-0.8 \mu \mathrm{m}$ apart at the time of coculture, the BSTp explants often appeared to retract and form palisade-like structures (arrow) along the border facing the AVPV explants after $10 \mathrm{~d}$ in vitro; this was seen only in female-female cocultures. $C$, When BSTp explants derived from female rats on P5 were cocultured with an AVPV explant derived from an androgen-treated female rat (P9), the density of neurites extending from the BSTp explant to the AVPV explant was similar to that observed in female-male cocultures, indicating that the ability of sex steroids to influence the density of neurites between cocultured explants is independent of the genetic sex of the animal from which the BSTp was derived and wholly dependent on the neonatal hormonal history of the animal contributing the AV PV explant. Scale bar, $15 \mu \mathrm{m}$.

a nucleus, there may be a concomitant increase in the number of neurons that provide inputs in a way that is analogous to the target-dependent regulation of neuronal survival seen in neuromuscular systems (Hollyday and Hamburger, 1976; Hamburger, 1977; Williams and Herrup, 1988). Our results in the AVPV demonstrate that the action of testosterone on the number of neurons in a target region can remain quite distinct from hormonal regulation of target innervation. Such target-dependent developmental mechanisms provide for a great deal of flexibility in how sex steroids sculpt the architecture of forebrain pathways involved in coordinating reproductive function. For example, the BSTp also provides direct projections to the medial preoptic and ventromedial nuclei of the hypothalamus, which play key roles in regulating expression of copulatory behavior (Hansen et al., 1982; Meisel and Sachs, 1994; Pfaff et al., 1994), yet the projections to these nuclei are much less dimorphic than those to the AVPV (Gu et al., 1997; G. Gu, E. K. Polston, and R. B. Simerly, unpublished observations). It remains to be shown whether these region-specific differences in the density of innervation by BSTp neurons are attributable to differences in the projections of subpopulations of BSTp neurons that are differentially sensitive to the developmental actions of sex steroids or are attributable to sex-specific alterations in the architecture of neuronal collaterals. The functional outcome of this target specific sculpting of forebrain neural architecture may be that the same sensory cue can have profoundly different effects on distinct aspects of reproductive function.

The cellular mechanisms underlying target-dependent sexual differentiation of the BSTp to AVPV pathway remain unknown. A consistent finding in our coculture experiments was that the density of neurites extending between the pairs of explants was dependent on the orientation of the BSTp explant. Placement of the lateral side of the BSTp explant proximal to the AVPV explant resulted in only a few neurites extending toward the AVPV. In vivo, axons that project from the BSTp to the AVPV display a similar directionality by preferentially passing ventromedially through the preoptic region (Hutton et al., 1998). In cocultures, at least a few neurites did extend outward from the distal side of the BSTp explants, but consistently there were many more neurites emanating from the proximal side of the BSTp explants than from the distal side. Thus, neurons located along the medial edge of the BSTp explants appear to be particularly active in providing neurites that extend toward the AVPV. One possible explanation is that in males, the AVPV exerts a trophic effect on the BSTp such that there is preferential survival of BSTp neurons proximal to the AVPV explant that extend active neurites. However, it is unlikely that the differentiation in target innervation by BSTp neurons we observed is attributable to autonomous cellular events localized to the BSTp, because there was a dramatic difference between the density of neurites extending to the AVPV in male-male cocultures compared with that observed in male-female cocultures. Notably, we did not detect significant differences in the density of neurites that exit BSTp explants derived from male or female rats in any of the cocultures but observed a marked difference in the density of labeled fibers that reach the AVPV. Another interesting possibility is that the greater density of neurites observed in the region immediately adjacent to the AVPV in male-male cocultures is attributable to an increase in branching as the neurites approach the AVPV (Kalil et al., 2000). This interpretation is consistent with our data, but confirmation will require morphometric analysis of individual neurites or time-lapse imaging of fibers during neurite outgrowth. Nevertheless, such a mechanism implies that a target-derived factor is exerting a trophic action on BSTp neurons to promote branching in the region of the AVPV in a manner that is independent of the source of the BSTp neurons.

Because the density of neurites that extend to the AVPV is dependent on exposure of the AVPV tissue to testosterone and is independent of whether the BSTp explant is derived from a male or female rat, the most plausible interpretation of the present in vitro findings is that the AVPV exerts a tropic effect on the development of projections from the BSTp. Moreover, the BSTpAVPV cocultures consist of explants separated by $\sim 500-800 \mu \mathrm{m}$ in a collagen matrix, suggesting that this tropic activity is medi- 
ated by a diffusible factor, and a high density of neurites between explants fails to form if the explants are separated by $>1.5 \mathrm{~mm}$. This tropic activity also appears to function during a restricted developmental window, because neurite density between explants is much less in cocultures containing AVPV explants derived from rats younger or older than $\mathrm{P} 9$. Thus, it seems reasonable to propose that the sexual differentiation of the BSTp-to-AVPV pathway is a target-dependent developmental event mediated by the hormonal induction of chemotropic factors that act specifically on BSTp neurons during a defined postnatal critical period.

Although it is clear that axons can be directed to their targets by contact-mediated guidance cues (Sanes, 1989; Reichardt and Tomaselli, 1991), a rapidly expanding body of evidence supports the concept that diffusible factors are secreted by target neurons and either attract or repel afferent axons (Goodman, 1996; Mueller, 1999; Song and Poo, 1999). The ability of testosterone to induce a target-derived activity that causes sexual differentiation of the BSTp-to-AVPV pathway may be attributable to either attractive or repellent chemotropic factors. Thus, higher levels of testosterone present in newborn males may induce expression of chemoattractant substances by AVPV neurons that promote developing axons to innervate the AVPV. Alternatively, exposure to testosterone during neonatal life may permanently reduce expression of chemorepellents by AVPV cells, leading to enhanced innervation in males. Molecular factors governing the development of sexually dimorphic pathways remain unknown, but developmental cues influencing the sexual differentiation of the BSTp-to-AVPV pathway may be target-derived factors such as neurotrophins or secreted chemotropic molecules such as members of the netrin or semaphorin families (Colamarino and Tessier-Lavigne, 1995; Ming et al., 1997; Song et al., 1997; Raper, 2000; Tucker et al., 2001). A great deal of progress has been made recently in identifying chemotropic molecules and determining signaling mechanisms that direct formation of neural connections (Tessier-Lavigne and Goodman, 1996; Dodd et al., 1998; Flanagan and Vanderhaeghen, 1998; Mueller, 1999; Brose and TessierLavigne, 2000), but none of these has been shown to be regulated by sex steroid hormones. The testosterone-induced targetdependent activity that appears to direct masculinization of the sexually dimorphic projection from the BSTp to the AVPV may present a unique opportunity to identify such factors.

\section{REFERENCES}

Annis CM, Edmond J, Robertson RT (1990) A chemically-defined medium for organotypic slice cultures. J Neurosci Methods 32:63-70.

Arai Y (1981) Synaptic correlates of sexual differentiation. Trends Neurosci 4:291-293.

Arnold AP, Gorski RA (1984) Gonadal steroid induction of structural sex differences in the central nervous system. Annu Rev Neurosci 7:413-442.

Arnold AP, Jordan CL (1988) Hormonal organization of neural circuits. In: Frontiers in neuroendocrinology, Vol 10 (Martini L, Ganong WF, eds), pp 185-214. New York: Raven.

Barraclough CA (1979) Sex differentiation of cyclic gonadotropin secretion. In: Advances in the biosciences, Vol 25 (Kaye AM, Kaye M, eds), pp 433-450. Oxford: Pergamon.

Bleier R, Byne W, Siggelkow I (1982) Cytoarchitectonic sexual dimorphisms of the medial preoptic and anterior hypothalamic areas in guinea pig, rat, hamster, and mouse. J Comp Neurol 212:118-130.

Bloch GJ, Gorski RA (1988) Estrogen/progesterone treatment in adulthood affects the size of several components of the medial preoptic area in the male rat. J Comp Neurol 275:613-622.

Brose K, Tessier-Lavigne M (2000) Slit proteins: key regulators of axon guidance, axonal branching, and cell migration. Curr Opin Neurobiol 10:95-102.

Colamarino SA, Tessier-Lavigne M (1995) The axonal chemoattractant netrin-1 is also a chemorepellent for trochlear motor axons. Cell 81:621-629.

Cooke B, Hegstrom CD, Villeneuve LS, Breedlove SM (1998) Sexual differentiation of the vertebrate brain: principles and mechanisms. Front Neuroendocrinol 19:323-362.

Dani JW, Amstrong DM, Benowitz LI (1991) Mapping the development of the rat brain by GAP-43 immunocytochemistry. Neuroscience 40:277-287.

Dodd J, Jessell TM, Placzek M (1998) The when and where of floor plate induction. Science 282:1654-1657.

DonCarlos LL, Handa RJ (1994) Developmental profile of estrogen receptor mRNA in the preoptic area of male and female neonatal rats. Dev Brain Res 79:283-289.

Flanagan JG, Vanderhaeghen P (1998) The ephrins and Eph in neural development. Annu Rev Neurosci 21:309-345.

Forger NG, Hodges LL, Roberts SL, Breedlove SM (1992) Regulation of motoneuron death in the spinal nucleus of the bulbocavernosus. J Neurobiol 23:1192-1203.

Gerall AA, Givon L (1992) Early androgen and age-related modifications in female rat reproduction. In: Handbook of behavioral neurobiology (Gerall AA, Moltz H, Ward IL, eds), pp 313-354. New York: Plenum.

Godement P, Vanselow J, Thanos S, Bonhoeffer F (1987) A study in developing visual systems with a new method of staining neurons and their processes in fixed tissue. Development 101:697-713.

Goodman CS (1996) Mechanisms and molecules that control growth cone guidance. Annu Rev Neurosci 19:341-377.

Gorski RA (1973) Perinatal effects of sex steroids on brain development and function. In: Progress brain research, Vol 39 (Zimmermann E, Gispen WH, Marks BH, de Wied D, eds), pp 149-163. Amsterdam: Elsevier.

Gu GB, Simerly RB (1997) Target specific hormonal regulation of sexually dimorphic projections from the principal nucleus of the bed nuclei of the stria terminalis. Soc Neurosci Abstr 23:341.

Guthrie S, Lumsden A (1994) Collagen gel coculture of neural tissue. Neuroprotocols 4:116-120.

Hamburger V (1977) The developmental history of the motor neuron. Neurosci Res Program Bull 15[Suppl iii]:37.

Hansen S, Köhler C, Goldstein M, Steinbusch HVM (1982) Effects of ibotenic acid-induced neuronal degeneration in the medial preoptic area and the lateral hypothalamic area on sexual behavior in the male rat. Brain Res 239:213-232.

Harris GW (1970) Hormonal differentiation of the developing central nervous system with respect to patterns of endocrine function. Philos Trans R Soc Lond [Biol] 259:165-177.

Hollyday M, Hamburger V (1976) Reduction of the naturally occurring motor neuron loss by enlargement of the periphery. J Comp Neurol 170:311-320.

Hutton LA, Simerly RB (1997) Influence of sex steroids on expression of estrogen receptor mRNA in explant cultures of the principal nucleus of the bed nuclei of the stria terminalis. Soc Neurosci Abstr 23:343.

Hutton LA, Gu GB, Simerly RB (1998) Development of a sexually dimorphic projection from the bed nuclei of the stria terminalis to the anteroventral periventricular nucleus in the rat. J Neurosci 18:3003-3013.

Ibanez MA, Zee J, Crabtree M, Simerly RB (1998) Developmental critical period for sexual differentiation of dopaminergic neurons in the anteroventral periventricular nucleus (AVPV). Soc Neurosci Abstr 24:1546.

Kalil K, Szebenyi G, Dent EW (2000) Common mechanisms underlying growth cone guidance and axon branching. J Neurobiol 44:145-158.

Kuang RZ, Merline M, Kalil K (1994) Topographic specificity of corticospinal connections formed in explant coculture. Development 120:1937-1947

Lee MK, Tuttle JB, Rebhun LI, Cleveland DW (1990) The expression and posttranslational modification of a neuron specific beta-tubulin isotype during chick embryogenesis. Cell Motil Cytoskeleton 17:118-132.

McAbee MD, DonCarlos LL (1998) Ontogeny of region-specific sex differences in androgen receptor messenger ribonucleic acid expression in the rat forebrain. Endocrinology 139:1738-1745.

McGuire CB, Snipes GJ, Norden JJ (1988) Light-microscopic immunolocalization of the growth- and plasticity associated protein GAP-43 in the developing rat brain. Dev Brain Res 41:277-291.

Meisel RL, Sachs BD (1994) The physiology of male sexual behavior. In: The physiology of reproduction, Ed 2 (Knobil E, Neill JD, eds), pp 3-105. New York: Raven.

Ming G, Lohof AM, Zheng JQ (1997) Acute morphogenic and chemotropic effects of neurotrophins on cultured embryonic Xenopus spinal neurons. J Neurosci 17:7860-7871.

Moody SA, Miller V, Spanos A, Frankfurter A (1996) Developmental expression of a neuron-specific beta-tubulin in frog (Xenopus laevis): a marker for growing axons during embryonic period. J Comp Neurol $364: 219-230$

Mueller BK (1999) Growth cone guidance: first steps towards a deeper understanding. Annu Rev Neurosci 22:351-388.

Pennypacker K, Fischer I, Levitt P (1991) Early in vitro genesis and differentiation of axons and dendrites by hippocampal neurons ana- 
lyzed quantitatively with neurofilament- $\mathrm{H}$ and microtubule-associated protein 2 antibodies. Exp Neurol 111:25-35.

Pfaff DW, Schwartz-Giblin S, McCarthy MM, Kow L (1994) Cellular mechanisms of female reproductive behaviors. In: The physiology of reproduction, Ed 2 (Knobil E, Neill J, eds), pp 107-220. New York: Raven.

Raisman G, Field PM (1971) Sexual dimorphism in the preoptic area of the rat. Science 173:731-733.

Raper JA (2000) Semaphorins and their receptors in vertebrates and invertebrates. Curr Opin Neurobiol 10:88-94.

Reichardt LF, Tomaselli KJ (1991) Extracellular matrix molecules and their receptors: functions in neural development. Annu Rev Neurosci 14:531-570.

Rinaman L, Card JP, Enquist LW (1993) Spatiotemporal responses of astrocytes, ramified microglia, and brain macrophages to central neuronal infection with pseudorabies virus. J Neurosci 13:685-720.

Sanes JR (1989) Extracellular matrix molecules that influence neural development. Annu Rev Neurosci 12:491-516.

Segovia S, Guillamón A (1993) Sexual dimorphism in the vomeronasal pathway and sex differences in reproductive behaviors. Brain Res Rev 18:51-74.

Shafit-Zagardo B, Kalcheva N (1998) Making sense of the multiple MAP-2 transcripts and their role in the neuron. Mol Neurobiol 16:149-162.

Simerly RB (1990) Hormonal control of neuropeptide gene expression in sexually dimorphic olfactory pathways. Trends Neurosci 13:104-110.

Simerly RB (1995) Hormonal regulation of limbic and hypothalamic pathways. In: Neurobiological effects of sex steroid hormones (Micevych PE, Hammer Jr RP, eds), pp 85-114. Cambridge, UK: Cambridge UP.
Simerly RB (1998) Organization and regulation of sexually dimorphic neuroendocrine pathways. Behav Brain Res 92:195-203.

Simerly RB (1999) Development of sexually dimorphic forebrain pathways. In: Sexual differentiation in the brain (Matsumoto A, ed). Boca Raton, FL: CRC.

Song HJ, Poo MM (1999) Signal transduction underlying growth cone guidance by diffusible factors. Curr Opin Neurobiol 9:355-363.

Song HJ, Ming GL, Poo MM (1997) cAMP-induced switching in turning direction of nerve growth cones [Erratum (1997) 389:412]. Nature 388:275-279.

Sumida H, Nishizuka M, Kano Y, Arai Y (1993) Sex differences in the anteroventral periventricular nucleus of the preoptic area and in the related effects of androgen in prenatal rats. Neurosci Lett 151:41-44.

Tessier-Lavigne M, Goodman CS (1996) The molecular biology of axon guidance. Science 274:1123-1133.

Tobet SA, Fox TO (1992) Sex differences in neuronal morphology influenced hormonally throughout life. In: Handbook of behavioral neurobiology, Vol. 11, Sexual differentiation (Gerall AA, Moltz H, Ward IL, eds), pp 41-83. New York: Plenum.

Toran-Allerand CD, Singh M, Setalo Jr G (1999) Novel mechanisms of estrogen action in the brain: new players in an old story. Front Neuroendocrinol 20:97-121.

Tucker KL, Meyer M, Barde YA (2001) Neurotrophins are required for nerve growth during development. Nat Neurosci 4:29-37.

Wiegand SJ, Terasawa E (1982) Discrete lesions reveal functional heterogeneity of suprachiasmatic structures in regulation of gonadotropin secretion in the female rat. Neuroendocrinology 34:395-404.

Williams RW, Herrup K (1988) The control of neuron number. Annu Rev Neurosci 11:423-453. 